\title{
Restrictions of production engineering on micro-specific product development
}

\author{
A. Albers, J. Marz
}

\begin{abstract}
The influences deriving from technology on the functions achievable by means of product design are typical characteristics of the development of micro-technological products. As a result, unlike the conventional design process the function attainable in micro technology due to product design appears to be less subjected to certain requirements. Multitechnological influences, resulting from production processes, material characteristics or micro-specific effects have an especially restrictive impact on the design of machine elements. On the basis of the product development process for primary shaped micro components from metallic and ceramic materials a method is presented which makes it possible to detect the know-how provided by disciplines subsequent and adjacent to the design process, having influence on the design of the machine element and interpret this relevant to design. The computer-aided availability of knowledge is accomplished with a knowledge-based design environment.
\end{abstract}

\section{1}

\section{Introduction}

Up to now the product development processes in micro technology have not been sufficiently identified and described concerning their specific characteristics. Currently, individual process steps and disciplines above all the production - are being examined separately according to their scientific standard. Apart from that also aspects of quality assurance and automated operation require approaches which are subject to a higher degree of integration. Considering the fact that in the near future an increasing demand for micro-technological products in middle and large scale quantities is expected

Received: 30 June 2003/Accepted: 12 November 2003

\author{
A. Albers, J. Marz ( $\square)$ \\ Institute of Machine Design and Automotive Engineering, \\ University of Karlsruhe (TH), \\ Kaiserstrasse 12, 76128 Karlsruhe, Germany \\ e-mail: marz@mkl.uni-karlsruhe.de
}

The work presented is supported by the Deutsche

Forschungsgemeinschaft within the collaborative research center (Sonderforschungsbereich) 499 "Development, production and quality assurance of primary shaped micro components from metallic and ceramic materials".

This paper was presented at the First International Colloquium "Micro Production" in February 2003. there is presently a lack of an integrated view on the product development process guaranteeing the consistency of the process chain of primary shaped micro parts. It is necessary to transfer process and technology characteristics of production preparation, production, and materials and specimen testing gradually into the early steps of product development. This is the task of product development methodology. For this purpose, the Institute of Machine Design and Automotive Engineering researches how the approaches, methods and tools for product development known from mechanical and precision engineering can be adapted and extended to micro-specific matters.

\section{2}

\section{Micro-specific product design}

Components and systems of micro technology are multitechnology products. Therefore, optimal, innovative micro systems can only be realized in an integration of technology, process and product development, material sciences and simulation, embracing all disciplines.

\section{1}

\section{Multitechnological influences on micro-compatible design}

Multitechnological influences in micro technology such as material anisotropy or limited possibilities for manufacturing micro structures due to unavailable tools (Fig. 1) lead to an alternation of the approaches for development and design known from macro technology. Consequently, unless it infringes elementary design know-how almost every design can be produced in accordance with the conventional process for mechanical engineering, though great technological and financial efforts will be involved. In micro technology the designer frequently has to deal with the question which designs actually can be produced and which kind of behavior the product will show. In this context it is difficult to assess the large amount of expertise, separate it according to its relevance to product design and project it on the current development task.

\section{2}

\section{Restrictions of production technique}

In micro technology the boundary conditions concerning production technology have a considerable effect on the liberty of designing the system components. Thus in order to design an efficient micro component, which ideally fulfils the required function the designer needs to be able 


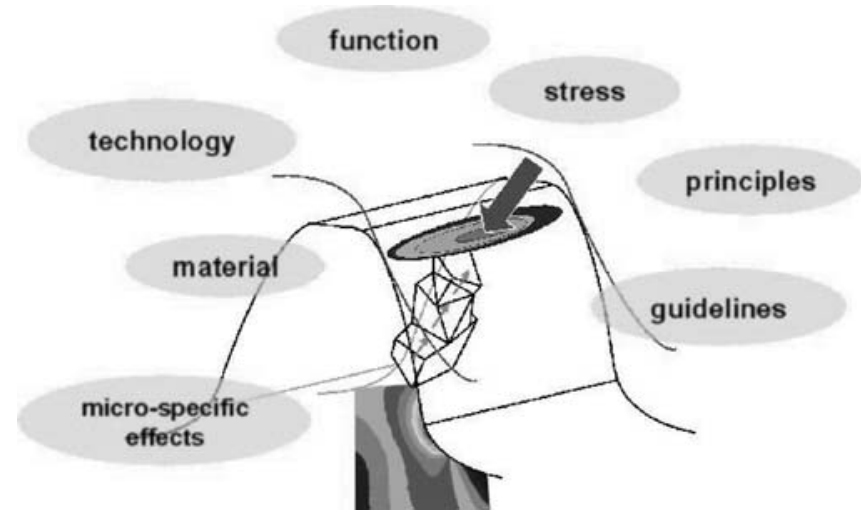

Fig. 1. Multitechnological boundary conditions influencing the micro-oriented design

to develop and design under conditions compatible to micro production.

Having realized that influences from production techniques are to be expected, technology specification tables were created (Fig. 2) in order to systematically collect the characteristic geometric dimensions and tool properties of production facilities and the manufacturing sizes achievable by using them (e.g. structure width, aspect ratio). In this context a distinction is made between production preparation and molding as the two essential production steps in the manufacturing of primary shaped metallic and ceramic micro components.

\subsection{1}

\section{Restrictions of production preparation}

The process of primary shaping firstly requires the manufacturing of a mold insert. With the aims to establish an efficient process chain for industrial large-batch and medium-sized production and to provide heavy-duty mi- cro system components by means of metallic and ceramic materials, abrasive and machining methods offer big advantages. Up to now the center of manufacturing mold inserts has been micro milling with micro end mill cutters.

The body diameter of the milling cutter as well as the length of the milling cutter's edge are process-specific characteristics, which have a restrictive effect on the part design. Apart from that also the tolerances of the machine tool, the tool and the process management are important. Moreover, phenomena such as formation of burrs and wear of tools are to be considered - as long as the latter cannot be compensated for by the process. Figure 2 shows an extract of the technology specification table for the micro milling process.

\subsection{2}

\section{Restrictions of molding}

The molding processes include the micro casting as well as the injection molding of micro-powder ( $\mu \mathrm{PIM})$, which can be distinguished in CIM for ceramics and MIM for metal. The $\mu$ PIM process is especially suitable for the aforementioned large-batch and medium-sized productions, since in these cases one can injection mold directly into the mold insert and at the same time manufacture a large number of micro components. The first thing required for the micro casting are models made of e.g. plastic, which one should be able to encapsulate. In this context deadmold casting covers specific fields of application.

Boundary conditions from the $\mu$ PIM derive from the necessity for runners, a sufficient number and size of part surfaces and points of attack for ejector pins used for demolding. The maximum yielding points and aspect ratios as well as sharp cross section transitions or bendings limit the mold-filling or the standard of the mold-filling technique. The shrinking, which occurs depending on the selected material, and which is almost

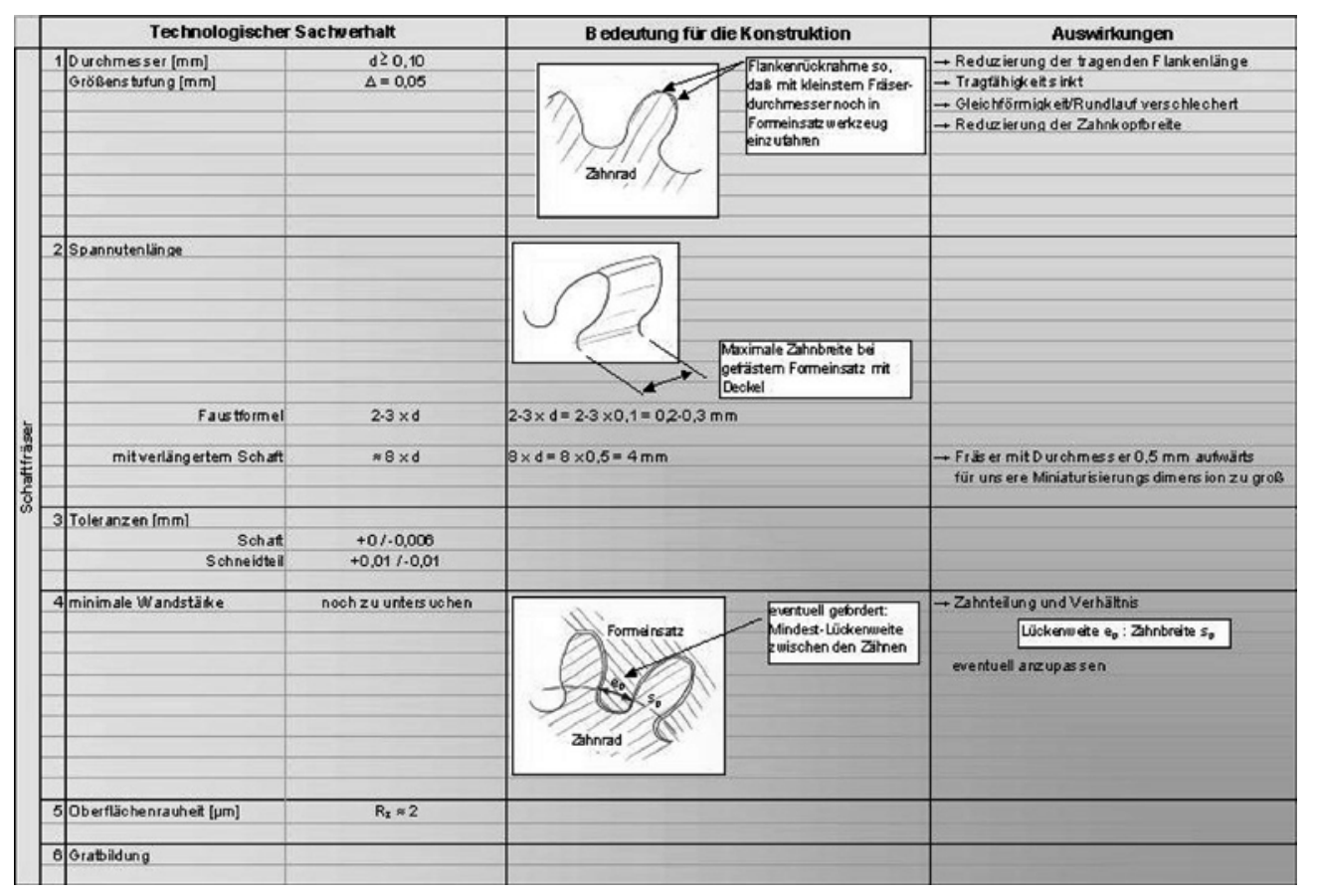

Fig. 2. Achievable manufacturing sizes in production preparation 
linear during the sintering process, should be particularly taken into account.

If the influence of the up to now collected technology data on the part design is also known, it is still the product designer himself, who has to carry out the projection on structural characteristics at micro components by means of his awareness of problems and his experience. In order to include process data and information effectively and regardless of individual people into the design process, the restrictions have been interpreted relevant to design. As a methodical aid design rules have been defined and made available by computer.

\section{3}

\section{Design rules - restrictions interpreted relevant to design}

Design rules are detailed instructions for a micro-compatible part design. They are derived from multitechnological basic requirements.

Depending on their degree of concretion design rules are suitable for different phases of product development.

In the process steps of product planning and conception they guide the designer by mentioning restrictive characteristics of his decision-making process. By means of translating quantitative process parameters into geometric mathematical expressions the knowledge becomes available for administration in databases and due to the connection to the design system also directly for supporting the embodiment design within the 3D CAD.

\section{1}

\section{Deriving design rules}

Figure 3 shows the steps for deriving design rules from multitechnological boundary conditions. The method is divided into the main sections of technology- related deriving of knowledge relevant to design and its computeraided availability during the process.

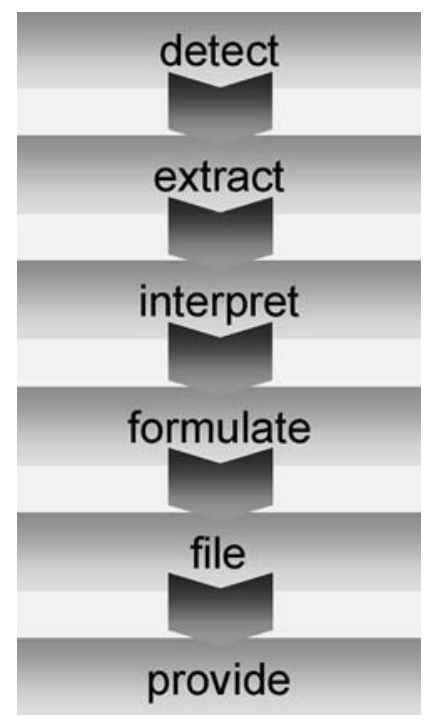

Fig. 3. Steps for deriving design rules from multitechnological boundary conditions
In Sect. 2 the detection of potential influences from existing production engineering technologies as well as the extraction and the interpretation of knowledge relevant to design have been presented in the form of technology specification sheets. However, before a computer-aided filing and availability can be realized the rule knowledge needs to be formulated in an appropriate way and structured according to characteristic criteria. As a result, the storage in and the access via a database system as well as the direct implementation of the rule knowledge into the design environment of a 3D CAD system becomes possible.

\section{2}

\section{Structure of rule presentation}

The structure of the presentation and an unambiguous identification of design rules has been accomplished with an especially defined classification scheme consisting of the rule class and a number (Fig. 4).

The rule class contains the type of machine element the rule refers to. Depending on the rule information concerning production technology and details about the tool and material group are given. If a rule can be applied to various production techniques or tool and material groups, the entries " $x x x$ " or " $x$ " are made instead of the listed code letters. However, this is not possible for the machine element type, as the contents of the rule has to be clearly assigned to the mold insert or the model. Therefore, there are generally different records of design rules for mold and model, which are nevertheless clearly connected geometrically. Restrictions from the mold insert manufacturing automatically affect the model and vice versa. However, a difference can be found in the geometrical properties. The model is scaled down due to a sinter shrinking during the molding process. Moreover, complementary figures are developed since a drilling in the mold insert results in a cylinder of lower height and diameter in the model. Thus, distinguishing according to the machine element type leaves it up to the product designer if he wants to develop the model of the primary shaped part or the corresponding mold insert.

\section{3}

\section{Examples of technology-related design rules}

The design rule KR_FE_MF3_x_002 is explained as an example.

It refers to the mold insert as machine element type and is valid for the production preparation technology of the 3 -axle micro milling. The entry " $\mathrm{x}$ " shows its validity for several tool groups (end mill and radius form cutters). Apart from that it is the second of its rule class composition. Expressed in a mathematical relation the rule is as follows:

$R_{\text {inner edge }} \geq \frac{d_{\text {milling cutter }}}{2}+T_{\text {mill }}$

Due to the circular cross section of the milling cutter no sharp inner edges or radii, which are smaller than the milling radius plus the milling tolerances, can be manufactured. For the concrete geometrical layout of a micro gear tooth (Fig. 5) this leads to a tip rounding of minimum 


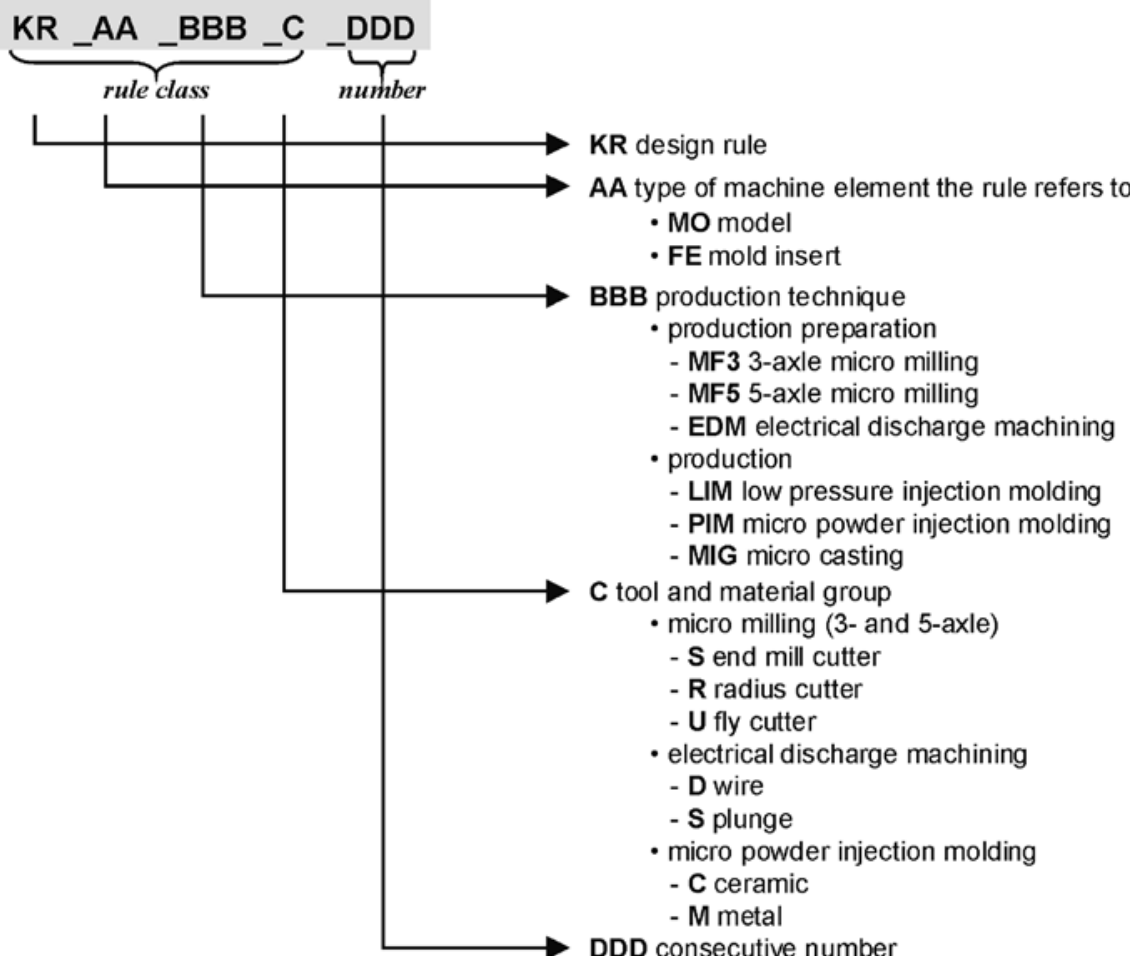

Fig. 4. Classification scheme for design rules

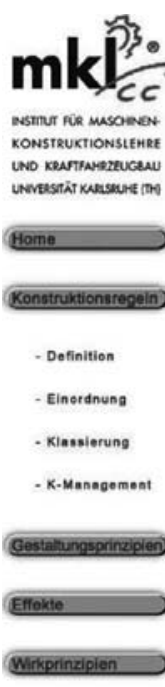

\section{Konstruktionsregeln}
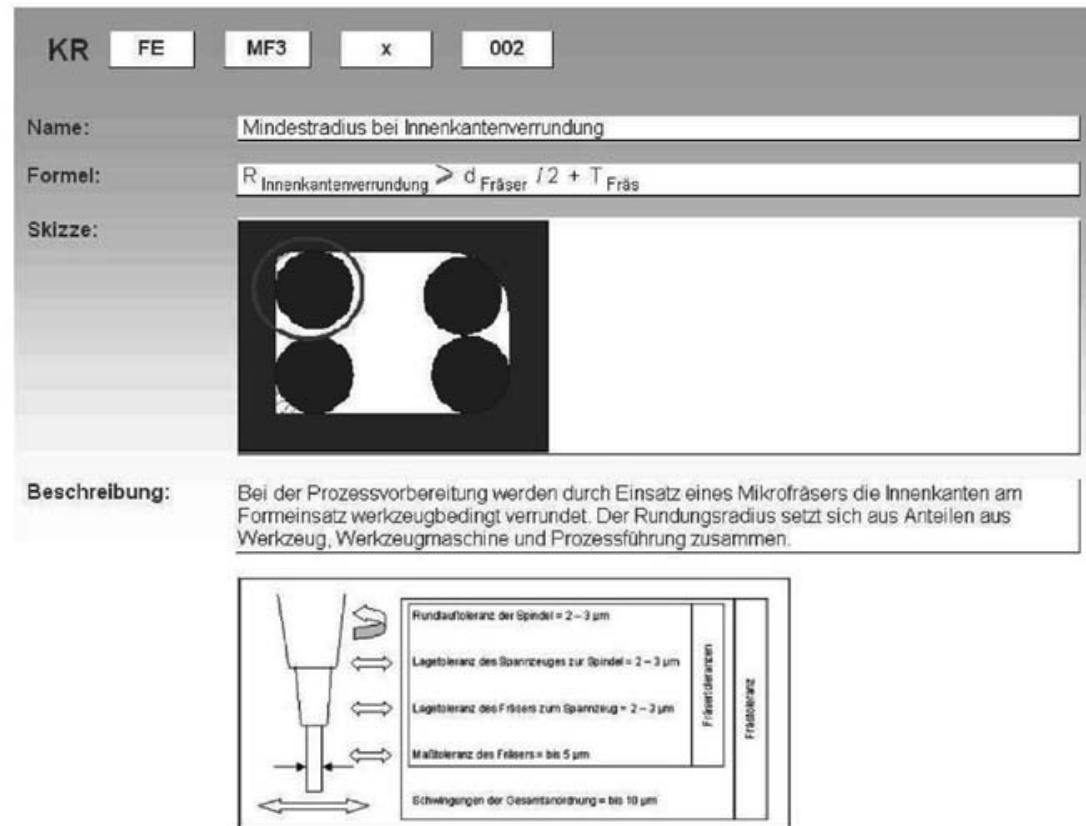

Graphik:

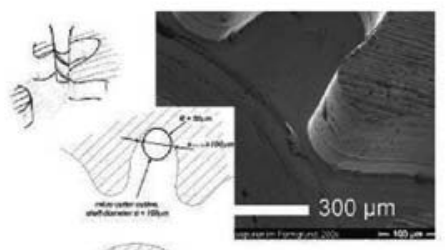




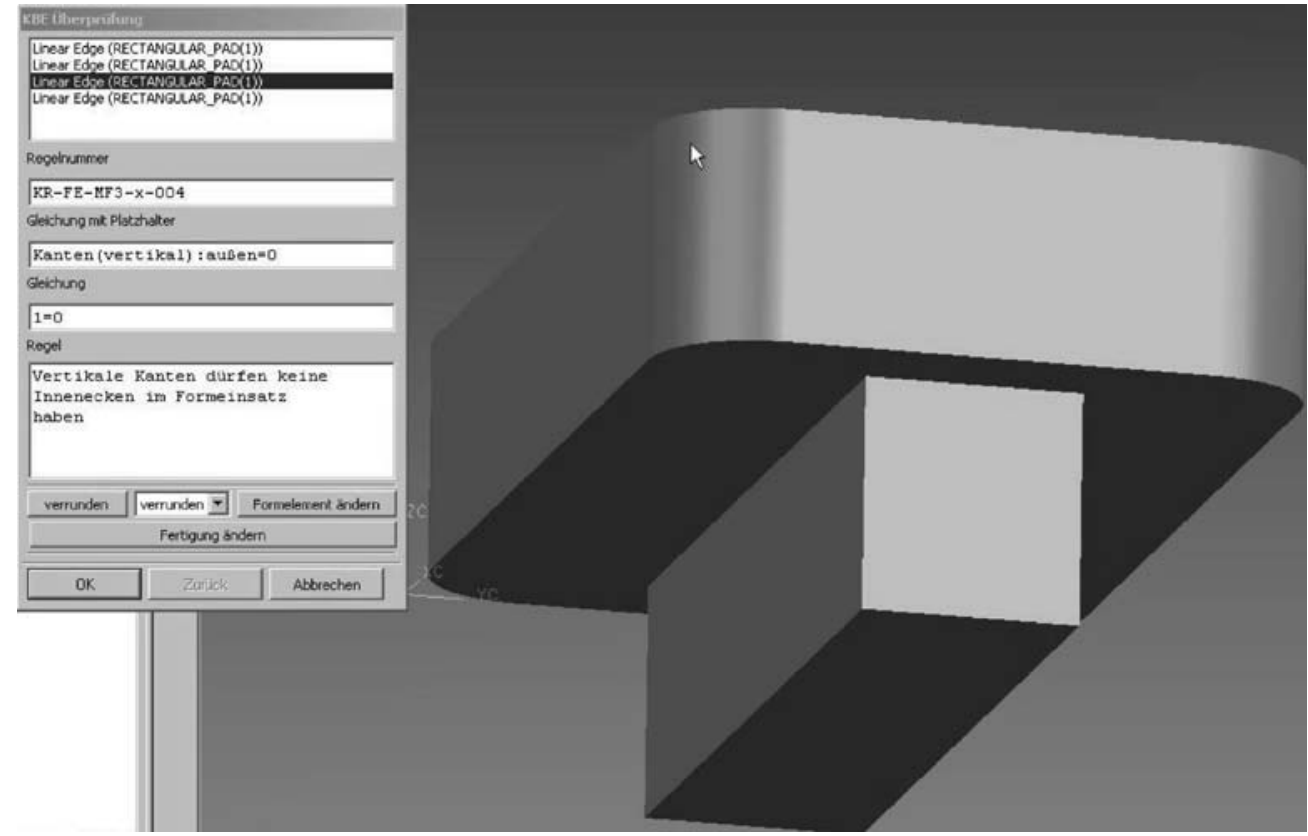

209

Fig. 6. Knowledge-based embodiment design (interactive window with infringed rules)
$50 \mu \mathrm{m}$ for the up to now smallest for reproducible cutting results utilized micro end mill cutter with a body diameter of $100 \mu \mathrm{m}$. This causes a loss of active flank length resulting in a declined transverse contact ratio which is essential for function requirement fulfilment.

\section{4}

\section{Computer-aided availability of design rules}

For a computer-aided availability of design rules the constitution of a knowledge-based design environment was approached. Both, an information unit and a threedimensional CAD system with an application for knowledge-based engineering (KBE) are integrated, accessing an unique database.

\section{1}

\section{The information unit: design and methodology database (KoMeth)}

"KoMeth" is an interactive knowledge portal which supports the product designer with specific design rules through different methods of access. For each design rule there exists an information site presenting the rule class and number, the title and the mathematical relation. Drafts, descriptions and pictures of virtual product models and manufactured components give illustrations (Fig. 5).

\section{2}

\section{The embodiment design unit: application for KBE within the 3D CAD system Unigraphics V18.0}

To apply design rules during the embodiment design phase, the application for knowledge-based engineering (KBE) within the three-dimensional CAD system Unigraphics V18.0 [4] had to be upgraded by a specific range of functions.

Starting a CAD session the designer is requested to define the type of machine element and details about the tool and material group. The programmed algorithm extracts edges and surfaces from the design draft with respect to the method of boundary representation - B-rep. [3] and relations between the boundary elements. The detected geometrical properties are connected together with the corresponding design rules, which have been concretised by parameters of the production techniques, resulting in elementary rules. These elementary rules represent mathematical relations that can be applied to the design draft by means of the application UG/KF (Unigraphics/Knowledge Fusion). Any infringement of a rule is indicated in an interactive window (Fig. 6).

\section{5}

\section{Conclusions}

Restrictions of production engineering were presented with respect to their influence on the product development process for primary shaped micro components from metallic and ceramic materials. By interpreting relevant to design they expand into the micro-production-oriented development and design in the form of design rules.

The design rules can particularly be classified along the type of machine element they refer to and the manufacturing technology and stored in a database.

Their computer-aided availability was realized by the interactive knowledge portal "KoMeth" on the one hand and by dint of the knowledge-based application within the 3D CAD system Unigraphics on the other. In future, both units will be increasingly integrated in a knowledge-based design environment.

\section{References}

1. Albers A; Burkardt N; Marz J (2003) Restrictions in the design of gear wheel components and drives for micro technology. Microsyst Technol 9: 192-196. DOI: 10.1007/s00542-0020213-7

2. Albers A; Marz J; Burkardt N (2002) Entwicklungsmethodik bei Konzeption und Entwurf eines Mikro-Planetengetriebes. Proceedings of "47. Internationales Wissenschaftliches Kolloquium”, Technische Universität Ilmenau 
3. Cherian RP; Midha PS; Smith LN; Pipe AG (2001) Knowledge 4. Unigraphics (2002) CAD/CAM/CAE-System für die durchbased and adaptive computational techniques for concurrent design of powder metallurgy parts. Adv Eng Software 32: 455465 gängige virtuelle Produktentwicklung (VPD) http://www.ugsolutions.de/produkte/unigraphics.shtml 